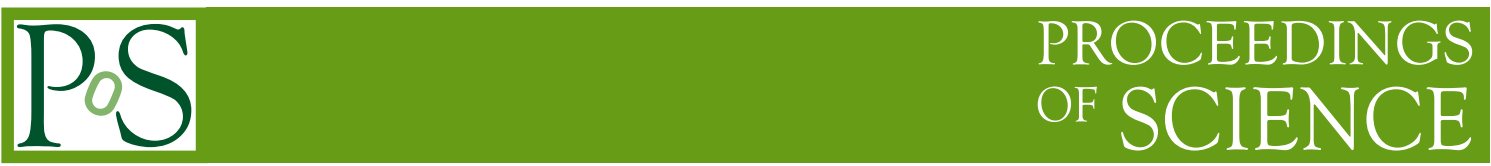

\title{
STRAW: STRings for Absorption length in Water
}

\author{
C. Fruck*, I.C. Rea, for the STRAW Collaboration ${ }^{\dagger}$ \\ Physics Dept., Technical University Munich, Germany \\ E-mail: cfruckeph.tum.de, imma.rea@tum.de
}

\begin{abstract}
Neutrino astronomy uses large volume detectors to search for astrophysical neutrinos and pinpoint their origin. Detectors such as IceCube at the Geographic South Pole, the Gigaton Volume Detector (GVD) in Lake Baikal and KM3NeT in the Mediterranean sea, instrument up to a cubic kilometer of water or ice for measuring Cherenkov radiation created in neutrino-matter interactions. Especially the utilization of the clear water of the deep sea as Cherenkov medium, in the past, has been facing severe difficulties in deploying and maintaining the offshore infrastructure. Ocean Networks Canada (ONC), an initiative of the University of Victoria, has been operating deep sea infrastructure for scientific purposes, off the Canadian coast since years. One of their network nodes, located on the Pacific abyssal plain, off the coast of Vancouver Island - Cascadia Basin - could be an ideal site for a future neutrino telescope. The Strings for Absorption Length in Water (STRAW) were developed at the Technical University of Munich (TUM) in collaboration with ONC and the University of Alberta. Two strings with optical modules have been deployed at Cascadia Basin in order to measure the optical properties of the water and study the feasibility of a larger installation. We will give a brief overview of the STRAW setup and present first results on the absorption length and optical background at Cascadia Basin.
\end{abstract}

36th International Cosmic Ray Conference -ICRC2019-

July 24th - August 1st, 2019

Madison, WI, U.S.A.

\footnotetext{
*Speaker.

${ }^{\dagger}$ http: //inspirehep. net/record/1701337. For collaboration list see PoS(ICRC2019)1177
} 


\section{Introduction}

Using high energy cosmic neutrinos as messengers in astronomy has some unique advantages but at the same time presents major challenges to the antiparticle community, both closely related to the fact that neutrinos only participate in the weak interaction. Therefore they are ideal probes for hadronic interactions, even if looking into dense astrophysical environments but at the same time they are notoriously hard to detect. The only known strategy so far is to instrument huge volumes (cubic-kilometers) of transparent material with light sensors [12]. The first attempt towards such a detector was actually supposed to be constructed in the deep ocean. The Deep Underwater Muon and Neutrino Detection (DUMAND) project aimed for a depth of $4800 \mathrm{~m}$ in the Pacific Ocean off the Big Island of Hawaii [7]. The project was cancelled after facing numerous challenges with deep water deployments and operation. The first and until now only successful attempt to instrument one cubic kilometer was made in ice with the completion of the IceCube detector at the south pole [3], now operating smoothly since 10 years. The data collected by IceCube, among other mile-stone discoveries lead to the detection of the first non-stellar neutrino source $[4,5]$. More recently two new projects have been launched towards the final goal of instrumenting a cubic kilometer of sea or lake water: KM3NeT [6], in the Mediterranean Sea and Baikal-GVD [8] in Siberia. A new opportunity presents itself in form of the vast area of instrumented sea floor called the NEPTUNE observatory by Ocean Networks Canada (ONC). NEPTUNE consists of hundreds of kilometers of sub-sea cables for power and data communication in the northeast Pacific Ocean in front of Vancouver Island. Of particular interest for neutrino astronomy is the deep-sea infrastructure at Cascadia Basin ( $47^{\circ} 46^{\prime} \mathrm{N}, 127^{\circ} 46^{\prime} \mathrm{W}$ ), at a depth of $\sim 2600 \mathrm{~m}$ b.s.l. due to its favorable ocean floor topology, comparably slow currents and low sedimentation rate. In late 2017 the pathfinder mission "STrings for Absorption length in Water" (STRAW) was designed and built at the Technical University Munich (TUM) in collaboration with the ONC and the University of Alberta and deployed in June 2018 by an ONC vessel. A third mooring line, named STRAW-b, is presently under construction and will be deployed in Spring 2020. The design of a large volume neutrino telescope at Cascadia Basin has also began under the project name Pacific Ocean Neutrino Explorer (P-ONE).

\section{Design}

STRAW has been designed with all aspects of being a pathfinder mission for a large neutrino detector in mind. One of the main goals is to characterize the Cascadia Basin site regarding the optical properties of the water as well as backgrounds caused by ambient radioactivity and bioluminescence. Another goal is to face the challenges that come with deploying and operating photomultipliers (PMTs) in an array of strings equipped with optical modules at a depth of more than 2 kilometers below the water surface. Therefore it was decided to measure the attenuation length by emitting light flashes with an early version of the Precise Optical Calibration Module (POCAM) [13], initially designed for the IceCube upgrade. Those are then received by the STRAW Digital Optical Modules (sDOMs) that were newly developed, based on the design of the POCAM housing. The three light-emitter modules (POCAMs) and the five sensor modules (sDOMs), in combination form baselines of different lengths that range from $20 \mathrm{~m}$ to $90 \mathrm{~m}$ and also implement a certain degree of redundancy for safety against module malfunction and cross-checking (see Fig. 1). Each 


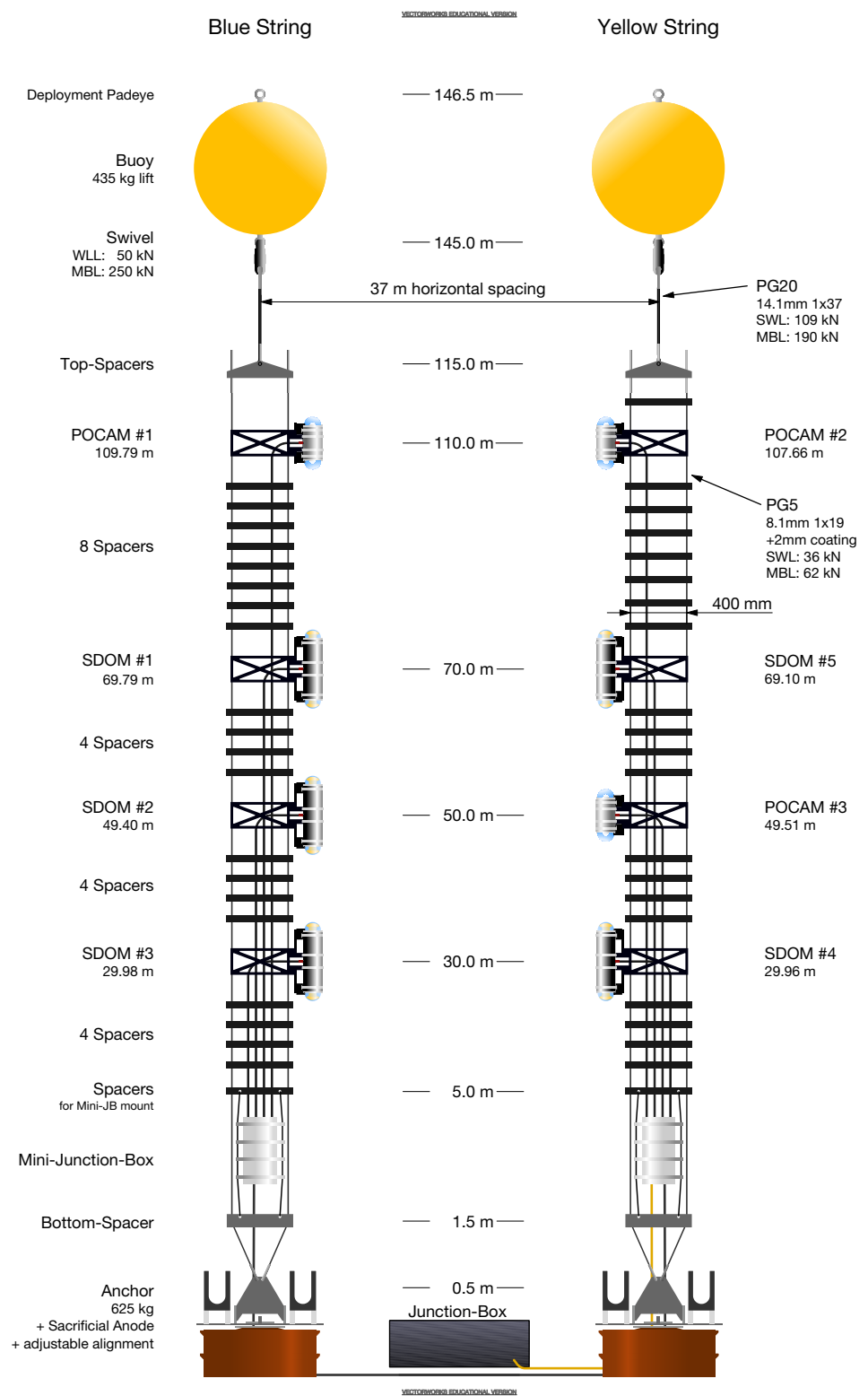

Figure 1: Detailed technical sketch of the two STRAW mooring lines showing the exact (measured) geometry of all modules.

string consists of two parallel steel lines of about $115 \mathrm{~m}$ length, separated by $30 \mathrm{~cm}$ wide metal spaces and stretched between a bottom anchor and a deep-sea buoyancy at the top. All electric and data connections are realized through sub-sea, multi-wire copper cables to each module that are attached to the spacers in a way that minimizes load on, and shadowing by the cables. The modules are mounted on one side of the strings to ensure a free light path between two neighbouring modules along one string. Additionally the two strings that have been installed at a relative distance of $37 \mathrm{~m}$ are equipped with rotatable platforms on the anchors that allow a remotely operated vehicle (ROV) to adjust the relative orientation of the strings until the modules face each other. All cables from the modules are connected to the Mini-Junction-Boxes (MJBs) in a star like configuration. 


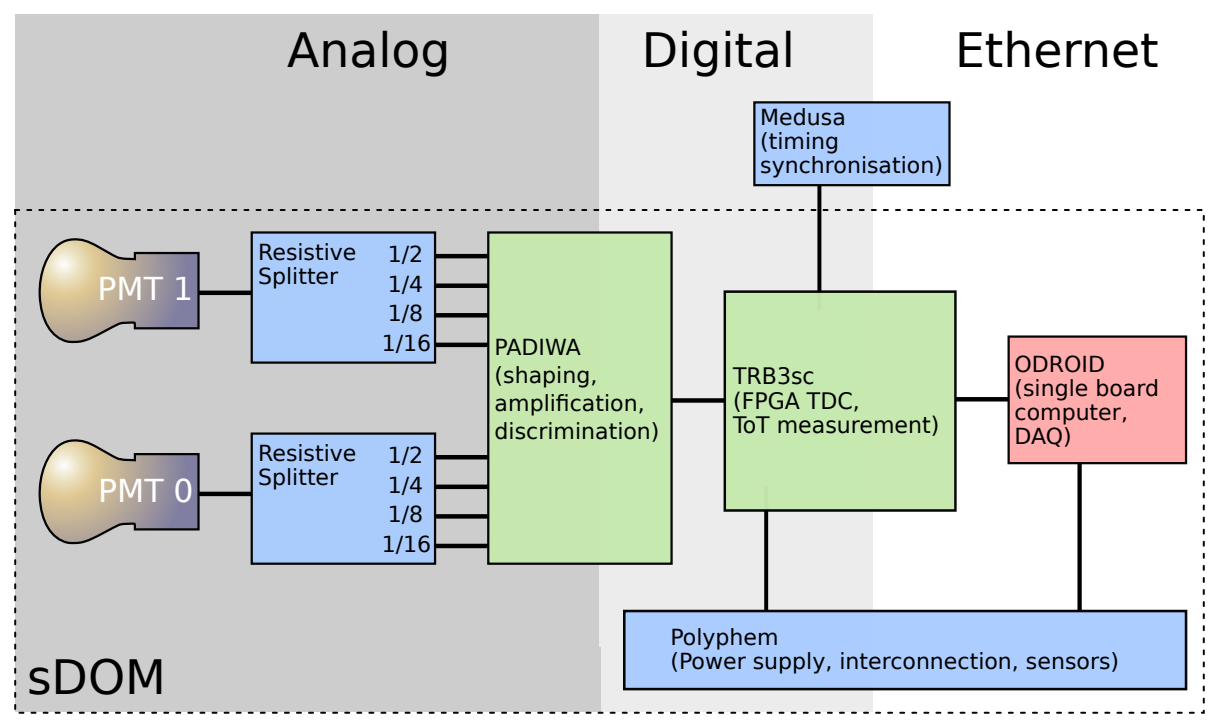

Figure 2: Schematic diagram of the sDOM electronics. The colors code parts the were produced in house (blue), provided by GSI (green), and bought off the shelf (red).

The MJBs in turn are daisy chained and connected to a main Junction Box of the ONC Neptune observatory using wet-matable ODI cables. The details of the mechanical structure are shown in Fig. 1.

The main functional element of the POCAM is an LED flasher with 12 channels (6 per hemisphere) using two different methods of producing short (ns timescale) light pulses. One is inspired by the one of J.S. Kapustinsky [11] and the other design directly uses the FPGA that forms the central element of the POCAM electronics by driving the LED with an amplified version of one of the FPGA output signals. Five different LED types with central wavelengths 365, 400, 465, 525 and $605 \mathrm{~nm}$ have been used. The light pulses generated using the faster Kapustinsky design have a fixed FWHM of $<10$ ns and emit $10^{9}$ photons, while the FPGA-driven pulses are $>40$ ns but adjustable in length and therefore allow for emitting even more intense flashes. Another main design feature of the POCAM is the isotropy of the emitted light pulse. This is achieved by diffusing the LED light using a semi transparent PTFE integrating sphere and by using two synchronized flashers that simultaneous emit light into two hemispheres that sum up to an overall isotropic profile with deviations only on the order of $5 \%$.

Each SDOM uses two R12199 PMTs, one glued inside the top and one inside the bottom hemisphere, with optical gel. Each PMT is connected to a resistive splitter with output ratios of 1/2, $1 / 4,1 / 8$ and 1/16 that connects the attenuated pulses into the readout system. The DAQ is based on a Trigger Read-out board (TRB3sc, [2]) with a PaDiWa as frontend card, both developed by GSI in Darmstadt. The PaDiWa takes care of shaping, amplification and discrimination, while the TRB3sc is operated as TDC unit for measuring arrival times and ToT. The system is sensitive to single photoelectrons with the least attenuated channel, while the channels with stronger attenuation only detect after-pulses and pulses with higher photon multiplicity. The readout is controlled via an Odroid C2 single board computer running DABC [1] on a Linux operating system. A schematic view of the sDOM signal chain can be viewed in Fig. 2. 
The sDOM units are operated as standalone instruments, each with it's own local DAQ, controlled via ssh connection over ethernet, but synchronized through a central timing system (CTS). The CTS is located inside one of the MJBs at the bottom of each string and can be used to reset the local clocks inside the sDOM TRBs.

\section{First measurements}

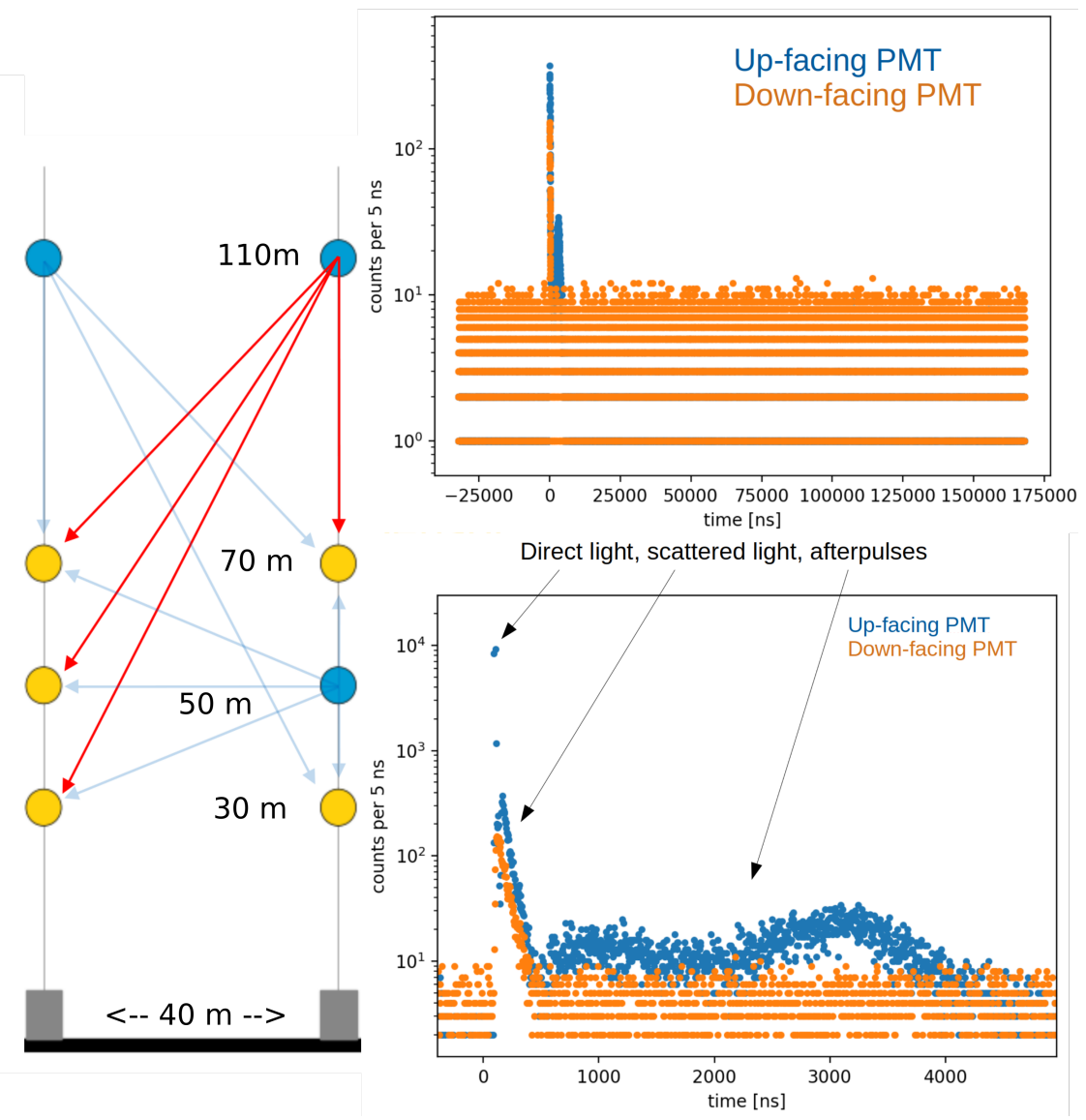

Figure 3: Illustration of the light attenuation measurement over different baselines with STRAW. The blue dots represent the POCAMs that act as precisely calibrated pulsed light sources and the yellow dots are the sDOMs that allow to measure the precise arrival time of photons on at the two PMTs (left side). A phasogram (histogram of the photon arrival times folded with the exact frequency of the flasher) reveals the photons emitted by the POCAM as a pileup of hits at a certain phase (upper right). A zoom-in onto the peak in the phasogram shows that the PMT facing the flasher receives mostly direct light, while the PMT facing away from the flasher only measures scattered photons that arrive at the detector taking detours of varying lengths (lower right). The phasogram also shows the after-pulsing distribution of the PMTs, between 0.5 and $4 \mu$ s after the direct peak.

The two main quantities STRAW was built for measuring are the light background at the bottom of Cascadia Basin and the attenuation length for Cherenkov light. In this section we describe our measurement techniques and show some preliminary results. The attenuation length $L_{T}$ can be 


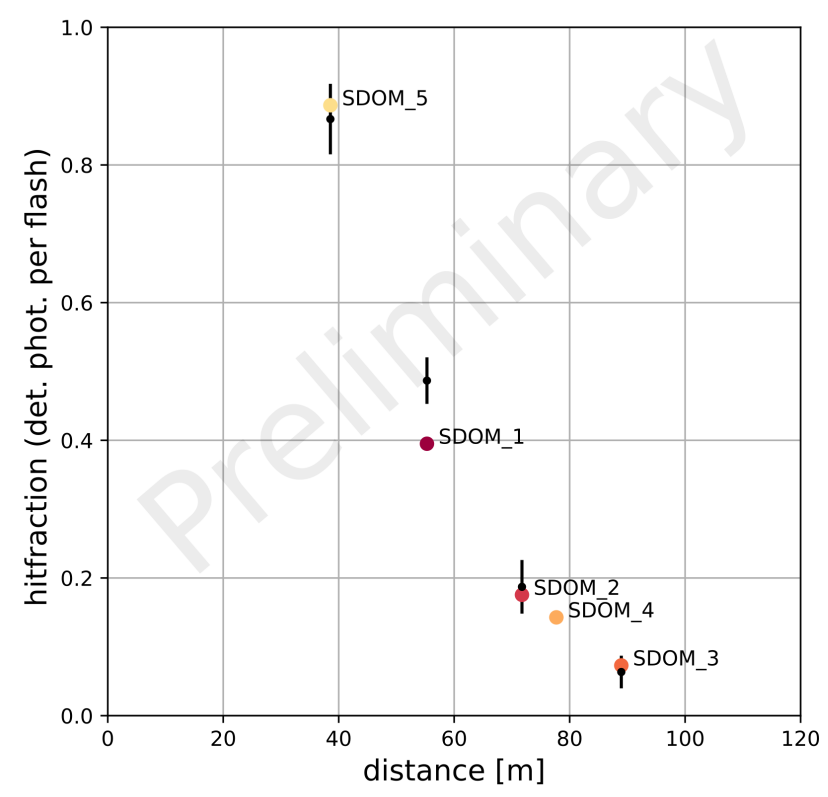

Figure 4: Measurement (black data points) compared to model prediction (colored dots) for the $465 \mathrm{~nm}$ POCAM LED at 12 V Kapustinsky bias.

determined through the following relation if $N_{0}$ is the number of initially emitted photons and $A_{\text {det }}$. is the detector area.

$$
N(r)=\frac{N_{0}}{4 \pi r^{2}} \exp \left(-\frac{r}{L_{T}}\right) A_{\text {det. }} .
$$

The geometry of STRAW allows that quantity to be measured at different distances $r$ between the light sources (POCAMs) and the detectors (sDOMs), as it is illustrated in Fig. 3 (left). The POCAMs are neither included in the timing system of the sDOMs nor do they emit an electronic trigger for the readout. They simply emit at a fixed predefined frequency on the order of a few $\mathrm{kHz}$ while the sDOMs are constantly recording the arrival times of all photons, including background from bioluminescence. In order to separate hits that can be associated with POCAM flashes from background events, a phasogram is generated by folding all time stamps into a single flasher phase and filling them into a histogram. This technique reveals a pileup of photons arriving with the exactly correct timing over a flat background from dark noise, radioactivity and bioluminescence (Fig. 3, right). The fraction of POCAM flashes resulting into a hit recorded with an sDOM can be compared to a model calculation that takes into account the geometry, angular emission/acceptance profiles and photon detection efficiencies, where the attenuation length is treated as a free parameter (see Fig. 4 for example). Uncertainties on the other parameters can be treated as nuisance parameters in a fit, a parameter scan or, as it has been done here, a MCMC sampling of the parameter space using the python package emcee [10]. First preliminary results for the four Kapustinsky driven LEDs are shown in Fig. 5. The second main purpose of STRAW is the measurement of the light background from bioluminescence and radioactive decays. This background can be measured with STRAW by constantly monitoring the rates in all 10 sDOM PMTs. The single photo-electron 


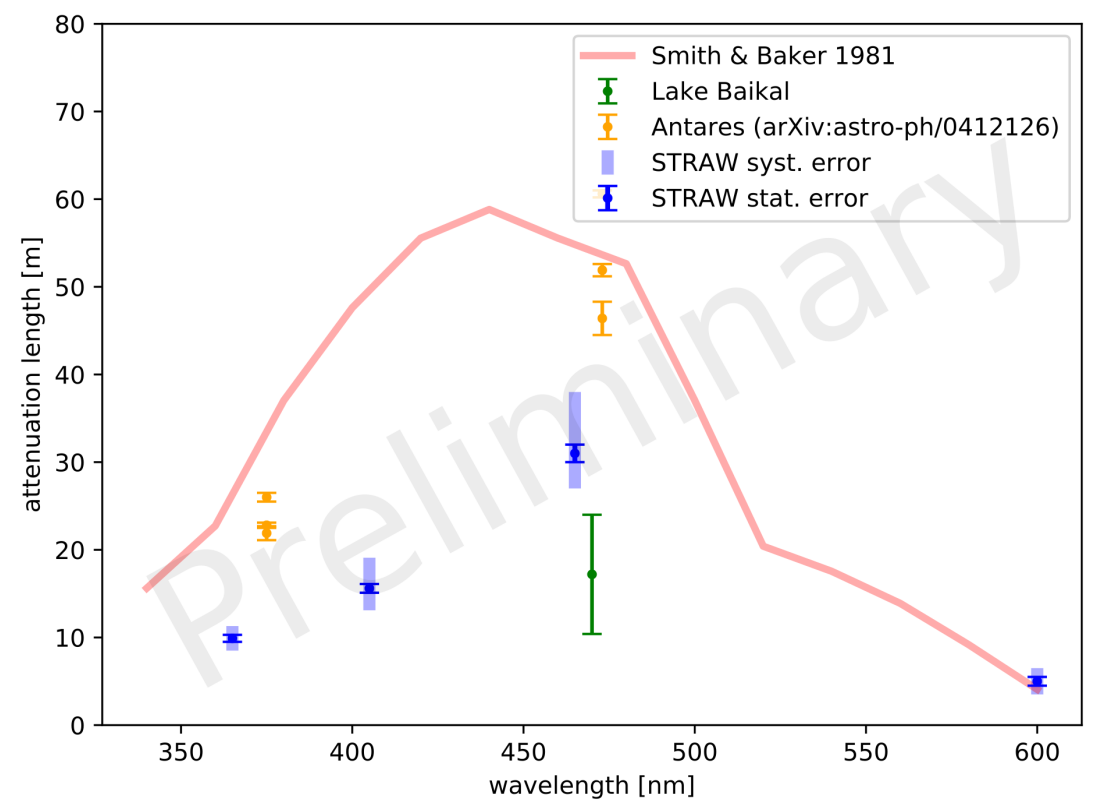

Figure 5: First preliminary result of the attenuation length measurements for the four LEDs that are connected to Kapustinsky flashers. The error bars show statistical and estimated systematic errors separately. Measurements in the cleanest sea waters [14] and for the ANTARES site [9] are given for reference.

rate for all PMTs is logged every $30 \mathrm{~ms}$ with close to $100 \%$ up-time and without changes to the measurement setup in terms of PMT HVs and discriminator thresholds since early 2019. Fig. 6 as an example shows the hourly average of the rates in all PMTs for the whole month of March. These data can be used for predicting the accidental trigger rate in a future neutrino telescope an thus for designing a multiplicity trigger that helps keeping this rate low enough, while still maintaining a reasonably low energy threshold. Apart from this main technical purpose, given the high time resolution and long up-time, they could also be used in a study of bioluminescent animals at the Cascadia Basin site.

\section{Summary}

We have presented the design and first measurement results of the STRings for Absorption length in Water (STRAW) experiment, the first of a series of pathfinder missions in the Pacific Ocean in front of Vancouver Island, Canada for evaluating the Cascadia Basin as possible future site of a large scale neutrino telescope. This site benefits form already existing infrastructure installed by Ocean Networks Canada, providing power and data connection ready to hook up. The two $120 \mathrm{~m}$ long strings, each equipped with five optical modules have been designed, built and deployed in less than one year. All data shown in this article have been recorded after full commissioning of the system, less then one year after the deployment. All data, despite only evaluated using a preliminary version of the analysis software, encourage further exploration of the site, including the planning of the first phase of a future neutrino telescope. 

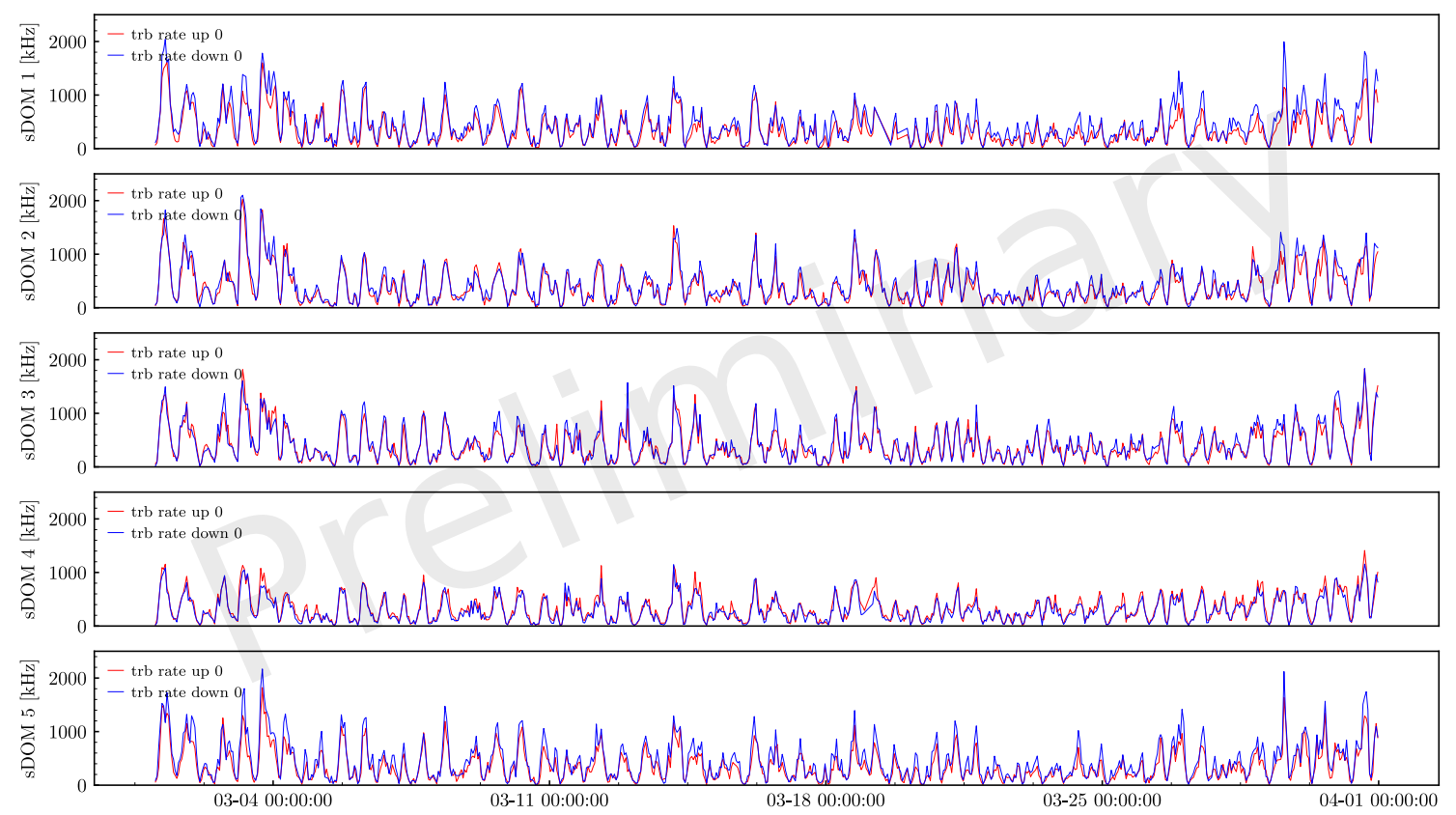

Figure 6: Hourly rates in all 5 sDOM for the whole month of March: (blue) down phasing PMT, (red) up phasing PMT. The nearly daily modulation observed is most probably due to the Ocean's tides. A correlation study is on-going.

\section{Acknowledgments}

The authors are grateful and appreciative of the support provided by Ocean Networks Canada, an initiative of the University of Victoria funded in part by the Canada Foundation for Innovation. In addition to their whole team, special thanks go to Jeannette Bedard and Ross Timmerman for assisting us in integrating STRAW into the ONC network infrastructure. This work is supported by the German Research Foundation through grant SFB 1258 "Neutrinos and Dark Matter in Astroand Particle Physics" and the cluster of excellence "Origin and Structure of the Universe".

\section{References}

[1] The Data Acquistion Backbone Core (DABC) framework. http://dabc.gsi.de/. Accessed: 09.10.2018.

[2] The TRB family of FPGA based readout boards. http://trb.gsi.de/. Accessed: 09.10.2018.

[3] M. G. Aartsen et al. The IceCube Neutrino Observatory: Instrumentation and Online Systems. JINST, 12(03):P03012, 2017.

[4] M. G. Aartsen et al. Multimessenger observations of a flaring blazar coincident with high-energy neutrino icecube-170922a. Science, 2018.

[5] M. G. Aartsen et al. Neutrino emission from the direction of the blazar TXS 0506+056 prior to the IceCube-170922A alert. Science, 361(6398):147-151, 2018.

[6] S. Adrián-Martínez et al. Letter of intent for KM3net 2.0. Journal of Physics G: Nuclear and Particle Physics, 43(8):084001, August 2016. 
[7] J. Babson et al. Cosmic Ray Muons in the Deep Ocean. Phys. Rev., D42:3613-3620, 1990.

[8] I. A. Belolaptikov et al. The Baikal underwater neutrino telescope: Design, performance and first results. Astropart. Phys., 7:263-282, 1997.

[9] ANTARES collaboration. Transmission of light in deep sea water at the site of the Antares neutrino telescope. Astroparticle Physics, 23(1):131-155, February 2005. arXiv: astro-ph/0412126.

[10] D. Foreman-Mackey, D. W. Hogg, D. Lang, and J. Goodman. emcee: The MCMC Hammer. , 125:306, March 2013.

[11] J.S. Kapustinsky et al. A fast timing light pulser for scintillation detectors. Nuclear Instruments and Methods in Physics Research Section A: Accelerators, Spectrometers, Detectors and Associated Equipment, 241(2):612 - 613, 1985.

[12] M. A. Markov and I. M. Zheleznykh. On high energy neutrino physics in cosmic rays. Nucl. Phys., 27:385-394, 1961.

[13] Elisa Resconi, Kai Krings Martin Rongen, Kai Krings, and IceCube-Gen2 collaboration. The Precision Optical CAlibration Module for IceCube-Gen2: First Prototype. In Proceedings of 35th International Cosmic Ray Conference - PoS(ICRC2017), page 934, Bexco, Busan, Korea, August 2017. Sissa Medialab.

[14] Raymond C. Smith and Karen S. Baker. Optical properties of the clearest natural waters (200-800 nm). Applied Optics, 20(2):177-184, January 1981. 